\title{
ÜBER EINIGE INNERSPRACHLICHE ZUSAMMENHÄNGE ZUR STEUERUNG VON SPRACHWANDEL
}

\author{
PIROSKA B. GERGELY
}

\begin{abstract}
Auszug
Heutzutage es ist allgemein verbreiteter Brauch in der historischen Sprachwissenschaft, dass diachrone Ergebnisse aus der synchronen Untersuchung der früheren Entwicklungsperioden der Sprache gezogen werden, um die Ursachen der linguistischen Veränderungen schlechterdings aufzudecken. Diese Arbeit versucht die Ursachen und den Prozess von morphophonologischen und morphosyntaktischen Veränderungen in der ungarischen Sprache sichtbar zu machen, die aus den inneren sprachlichen Zusammenhängen der mittel- und neuungarischen Perioden stammen und sich aus ihnen erklären lassen. Die untersuchten diachronen Erscheinungen gehören zu verschiedenen Änderungstypen. Sie können repräsentieren:

1. Systemzwang (wie im Falle des paradigmatischen Ausgleiches des Stammes idö 'Zeit');

2. Trennung von Form und Funktion (wie z.B. die diachrone Verbindung des Verbalpräfixes át- und der Postposition által 'durch') und

3. Wechselentwicklung der grammatischen Synonyme (wie z.B. Alternation der temporalen Bestimmungen mindéltig mindig 'immer').

Die Ursachen dieser Änderungen stammen aus dem synchronen System der gegebenen Periode und deuten darauf hin, dass allgemeine morphologische, semantische und funktionale Einflüsse ihres synchronen Verbindungssystems ihre historischen Veränderungen determinieren.
\end{abstract}

1. ,Synchronie ist die Schmiede der Diachronie“ — der Inhalt dieser weit verbreiteten und schönen wissenschaftlichen Metapher ist zur allgemeinen Methodik unserer sprachgeschichtlichen Forschungen geworden. Wenn die Möglichkeit gegeben ist, über den Sprachzustand vergangener Epochen eingehende Kenntnisse zu sammeln - unterstützt durch ein möglichst breites und vielfältiges sprachhistorisches Quellenmaterial —, so können wir entlang der Fäden der synchronen Zusammenhänge zu den meist komplexen Ursachen einzelner Sprachwandelphänomene gelangen, die sich aus den komplizierten Verflechtungen interner sprachlicher Zusammenhänge ergeben.

1216-8076/02/\$5.00 (C) 2002 Akadémiai Kiadó, Budapest 
In der vorliegenden Arbeit wird mit Hilfe der oben skizzierten Methode auf den Verlauf und die Ursachen einiger Wandelphänomene eingegangen, die sich zwischen dem 16-19. Jahrhundert abgespielt haben, in einer Zeit, aus der uns die meisten Daten der ungarischen Sprachgeschichte zur Verfügung stehen. Als Quelle dienten in erster Linie sprachhistorische und andere Wörterbücher, aber vor allem die üppigste Datenquelle dieser Epoche: Erdélyi magyar szótörténeti tár (= SzT., Historisches Wörterbuch des siebenbürgisch-ungarischen Wortschatzes).

2. Die Entstehung verschiedener Varianten des Stammes idô ('Zeit', ideje 'seine Zeit', idén 'heuer, dieses Jahr') sind gut belegt (Bárczi 1958, 36). Wir wissen auch, dass die auf -é auslautende Stammvariante im Altungarischen weiter verbreitet war als heute, sie trat auch in Formen auf, aus denen sie durch die Variante mit -ố verdrängt wurde. Diese Stammvariante lebt heute nur noch in den Wörtern idén 'heuer' und idétlen '(im Verhalten) unreif, in Form komisch, nicht gelungen' weiter, aber nur noch latent, da die Verbindung von ersterem zum Stammwort aufgrund der verblichenen Bedeutung von 'Jahr' in idô sich sehr gelockert hat, letzteres wiederum hat sich durch seine Bedeutung 'dumm, entstellt, unförmig' stark von der ursprünglichen Wortfamilie 'Zeit' isoliert.

In der mittelungarischen Zeit zeigt sich eine starke Schwankung innerhalb zweier Gruppen der Paradigmen der morphologischen Stammvarianten von idô. Die eine Gruppe besteht aus einer Reihe von Formen mit Possessivsuffix, wo neben den für die 3. Person bis heute gültigen Stammwechselformen (ideje 'Zeit + 3. P. Sg.' - idejük, mittelung. idejek 'Zeit + 3. P. Pl.') das Substantiv idố in der Bedeutung „Lebensdauer" in der 1. Person Singular und Plural häufig als Stammvariante mit -é vorkam (idém 'Zeit +1 . P. Sg.', idénk 'Zeit + 1. P. Pl.'), besonders im 16-17. Jh., vereinzelt auch noch am Anfang des 18. Jh. ${ }^{1}$

Beispiele: 1560: ,vagion ... wy haz kyt az en idemben wy helyre chinaltak" 'Es gibt ein neues Haus, das in meiner Zeit an neuer Stelle gemacht worden ist' (Néc SzD); 1568: „my Jdenkbe Jllyen kǫtest tǫttwnk hogy valaky ez dolgot haborgatna f. 20 maraggyon" 'Wir haben in unserer Zeit eine solche Verpflichtung auf uns genommen, dass wenn jemand diese Sache durcheinanderbringt, er 20 Forint Strafe zahlen soll' (Kv); 1668: ,az utan bujdosztanak el ..., en idemb(en) szarmasztanak vala haza" 'Sie sind danach in die Fremde gezogen ... in meiner Zeit kamen sie nach Hause' (Đés); 1754: „Mindenkor idémben

1 Die zitierten Belege sind dem SzT. entnommen. Nach dem zitierten Beleg folgt - hier und im Folgenden - in Klammern ein Verweis auf den Ort, zu den Abkürzungen vgl. SzT., Bd. 5, VII-VIII.

Acta Linguistica Hungarica 49, 2002 
tilalom alatt tartatott a nevezett Búk erdö“ 'In meiner Zeit stand der genannte Buchenwald immer unter Verbot' (Alfalu Cs).

Die andere Paradigmengruppe, in der die Stammvariante auf -é in derselben Epoche häufig vertreten ist, setzt sich aus den Ableitungen auf $-s$ in der Bedeutung 'alt, bejahrt' zusammen. Von ihren Vorkommen - auch in chronologischer Hinsicht — gibt in erster Linie das reiche Datenmaterial des SzT. Auskunft (im NySz. stehen lediglich einige literarische Belege aus dem 16. Jh.). ${ }^{2}$

Beispiele: 1561: „Az ides vín Embereknek az o allapattiok es ideiek zerent illendo tiztessegek megadassek“ 'Den betagten alten Leuten soll die Ehre nach ihrem Lebensalter und ihrer gesellschaftlichen Stellung gegeben werden' (Kv); 1575: „A hatarokra legien gongia eo kegnek lassak es Iaryak meg, ha my Igazgatas zwkseges benne ... vigenek Ides embereket kyk twgiak, Ifyakatis ky (!) Tanwlliak megh" 'Ihr sollt Euch um die Gemarkung kümmern, sie anschauen und begehen; sollte irgendeine Veränderung nötig werden, sollen alte Leute mitgenommen werden, die es wissen, und junge, die es lernen sollen' $(\mathrm{Kv})$; 1584: „Orsolia Istwan Kowachne Vgia(n) azont vallia, ezel teobiti hogy az Jdesbik wteotte le, az Iffiabbik ozta(n) vgy vagta“" 'Die Frau vom Schmied István Orsolya bezeugt dasselbe, fügt aber hinzu, dass der Ältere ihn/sie niedergeschlagen und der Jüngere ihn/sie erst dann geschlagen hat' (Kv); 1594: „Zabo Andras vallia ... Beleniesi Andras ... Baiuz Marton ... Idesbis nallamnal kez vagiok megh keouetnem" 'András Szabó sagt aus: András Belényesi, Márton Bajusz ist älter als ich, ich bin bereit, ihn um Verzeihung zu bitten' (Kv); 1603: „az Ides vraimban gywtessenek be tizen hatig Awagy huzigh valot, es az njolcz oztozo vraimnak egyenleo Akaratbol Jrianak Articulusokat" 'Von unseren alten Herren sollen sechzehn bis zwanzig zusammengerufen werden und für die acht sich in das Vermögen teilenden Herren auf gemeinsamem Einverständnis beruhende Beschlüsse schreiben’ (Kv); 1635/1650: „Vgian ezen Ceh giúlesekor vegesztek eo kglmek egesz Cehwl, Ides vraim mind ben leven“ 'In der Versammlung derselben Zunft wurde mit Einverständnis der ganzen Zunft der Beschluss gefasst, unsere alten Herren waren alle anwesend' (Kv); 1674: „Ides Uramékkal eo kglmekkel édszeris másszoris magunkis ittunk megh égy tiz vedres általagh ürmeos bort" 'Mit unseren alten Herren haben wir auch selbst ab und zu ein Fass zu zehn Eimern Wermutwein getrunken' (Kv).

Das Übergewicht der Variante idés 'alt' gegenüber idôs 'ds.' im 16. Jh. kann nicht für ein sprachgeographisches Phänomen gehalten werden - obwohl die relevanten Belege des SzT. alle aus demselben Ort: aus Kolozsvár (Klausen-

${ }^{2} \mathrm{Zu}$ den nächsten und den weiteren Belegen vgl. den Artikel idós, idósbik im SzT. Ein Teil der zitierten Belege bezieht sich auf die „Senioren“ des ehemaligen städtischen Großrats (centumviratus) bzw. der Zünfte. 
burg, Cluj, Rum.) stammen - , da diese Formvariante bereits aus dem Münchener Kodex (TESz.) und aus dem Érdy-Kodex (NySz.) bekannt ist. Im 17. Jh. überwiegt die Variante mit -ó, obwohl aus diesem Jahrhundert auch noch die Variante idés belegt ist (1674). Der analogische Ausgleich zugunsten von idôs scheint ab dem 18. Jh. endgültig zu sein, seit dieser Zeit kommt idés, idésb 'älter' im Beispielmaterial des NySz. oder des SzT. nicht mehr vor, nur noch idôs, idôsb 'älter'. Neben dem morphophonetischen Systemzwang kann als Ursache für den Wandel noch ein weiterer Aspekt herangezogen werden: die semantische Abkoppelung von idôs 'alt' und idétlen 'frühzeitig; kindisch'. Zwischen diesen beiden Ableitungen mit Privativum bzw. mit dem Ableitungssuffix -s 'mit etwas versehen' wird durch die semantische Opposition eine enge Verbindung geschaffen, die im Falle von idôs idés - idétlen besonders in der Zeit stark gewesen sein kann, als sich letzteres semantisch noch nicht von seinem Grundwort abgekoppelt hatte und in der Bedeutung 'vorzeitig, frühzeitig' verwendet wurde.

Diese Bedeutung kann in syntaktischen Verbindungen wie idétlen halál 'frühzeitiger Tod', idétlen kora 'js. zu junges, unreifes Alter', idétlen havak 'frühzeitiger, zu früher Schneefall', idétlen korán 'zu früh' (NySz.) noch im 1718. Jh. als lebendig nachgewiesen werden, obwohl die Weiterentwicklung dieser Bedeutung bereits früher, im späten Altungarischen eingesetzt haben kann, wie in Wortregistern aus dem 15. Jh. in solchen Wortfügungen wie idétlen gyermek 'frühes Kind', idétlen szülés 'frühzeitige Geburt' (RMGl.) sowie durch das erste Vorkommen des Wortes idétlen ohne syntaktische Ergänzungen im JókaiKodex belegt ist (TESz.). Durch die Bedeutung 'zu früh geboren, frühgeboren' wurde die semantische Verbindung von idés - idétlen bereits etwas gelockert, aber noch nicht aufgehoben. Die phonologische Gestalt idétlen, die meistens in der é-Variante vorkam, konnte also einen Einfluss auf die Lautgestalt der Ableitung idés mit dem Suffix -s ausüben, solange die semantische Opposition 'frühzeitig', 'zu früh' - 'alt' bestehen blieb. Als sich der Bedeutungswandel von idétlen allerdings fortsetzte und die Bedeutung 'frühzeitig', 'frühgeboren' immer mehr durch 'unentwickelt $\rightarrow$ formlos $\rightarrow$ unförmig' bzw. durch 'unreif $\rightarrow$ dumm' ersetzt wurde, hob sich die Opposition zu 'alt' und zugleich die stärkende Wirkung auf die Form idés auf. Nach der semantischen Ablösung von idétlen von der Wortfamilie idő übte der analogische Ausgleich eventuell einen stärkeren Einfluss aus, und die Form idés wurde durch idôs ersetzt. Die Position von idétlen wiederum, die nun auch keine formalen Verbindungen zu der ursprünglichen Wortfamilie mehr vorweisen konnte, wurde durch eine neue privativische Ableitung gefüllt: durch das paradigmatisch besser passende idótlen 'zeitlos'. 
Die Verdrängung der Stammvariante mit -é aus der Bedeutung 'alt' setzte sich in der Bedeutungssphäre 'Lebensdauer, Lebensalter' fort und kann auf die Abschaffung von idém, idénk '(in) mein(em) Leben, (in) unser(em) Leben' bzw. auf den Austausch durch die Varianten idôm, idônk gewirkt haben.

Der paradigmatische Ausgleich der Stammvarianten kann also bei weitem nicht auf eine einzige Ursache: auf die vereinende Wirkung des formalen Systemzwangs zurückgeführt werden, sondern erfolgt durch die Zusammenwirkung mehrerer Sprachschichten - wie am oben analysierten Beispiel gesehen - dann und in dem Teil des Paradigmas, wo konvergente Wirkungen aus den unterschiedlichen Sprachschichten eingetroffen sind.

3. Die morphologische Verselbstständigung mit Funktionsteilung sowie der durch synchrone Verbindungen gesteuerte Prozess der Bindung einzelner grammatischer Formvarianten zu grammatischen Funktionen und deren Befestigung kann in der Geschichte des Präfixes át 'durch (etwas hindurch)' und durch die Postposition által 'durch 〈Passiv〉' im 16-19. Jh. verfolgt werden. ${ }^{3}$

Im 16. Jh. verfügt nur die Postposition által über eine längere und eine kürzere Variante, wobei által in lokaler, temporaler und abstrakterer, vor allem instrumentaler Funktion gleichermaßen reich belegt ist, die kürzere Form ált ist jedoch zunächst durch eine einzige Quelle nachgewiesen, in der sie temporale Funktion einnimmt (1519 LányiK. TESz.). Ihr Fehlen in diesem reich belegten Jahrhundert lässt vermuten, dass ihre Frequenz gering war.

Das Präfix nahm in dieser Zeit ausschließlich die längere Form által (seltener átal) an, ihre mit zahlreichen Verben bestehenden Verbindungen haben meistens einen richtungsweisenden oder temporalen Bezug. 114 Verbindungen mit 86 Verben sind im 16. Jh. im NySz. sowie SzT. belegt.

Der Wandel, der letztendlich dazu führte, dass die längere Variante als Präfix verdrängt und - zumindest in der Schriftsprache - durch eine kürzere Form ersetzt wurde, setzte im nächsten Jahrhundert ein. Meines Wissens erscheint das Präfix erst jetzt in der Form ált, ihr erstes Vorkommen ist aus der Murányi Vénus (Venus von Murány) Gyöngyösis bekannt: 1663/64: ált-hattya 'durchdrängt', ált-kelte 'überqueren (Part.)', ált-ülte 'übersetzen (Part.)' (Simai 1910, 165), sowie aus dem 18. Jh., aus dem Sprachgebrauch József Gvadányis: ált-menvén 'überquert (Part.)', ált-hatott 'durchdrängt (Part.)', ált-húz 'durchzieht' (NySz.).

3 Zur Entstehungs- und Entwicklungsgeschichte der Postposition vgl. TNyt. I 450, 454; II/1 669-700, 704-7, 710-14; zu den Präfixen: I 436-37; II/1 664-66. 
Das Material des SzT. bezüglich des 18. Jh. ist äußerst reichhaltig, so dass bei der Untersuchung des Präfixes gegen Ende des Jahrhunderts auf mehrere hundert Belege zurückgegriffen werden kann. In den Belegen kommt ált kein einziges Mal, ausschließlich által vor. Eine so starke Übereinstimmung zwischen dem Zeugnis der Belege deutet darauf hin, dass der Status von által als Hauptvariante des Präfixes noch gegen Ende des 18. Jh. fest war, während die Kurzform ált eher eine seltene Ausnahme und eventuell lediglich eine stilistisch gebundene Variante war. Ihre stilistische Ebene war m. E. vermutlich näher an der gebildeten Litaratursprache als an der Umgangssprache angesiedelt. Dabei ist natürlich nicht die spärliche Zahl der Belege von Gyöngyösi bzw. Gvadányi, sondern gerade deren Abweichung von den Belegen des SzT. ausschlaggebend, das den eher umgangssprachlichen Sprachgebrauch von niederen Beamten und von im öffentlichen Leben Aktiven, Meistern, Landwirten usw. festhielt.

Es scheint daher sinnvoll, den Wandel des Präfixes im 19. Jh. kurz zusammenzufassen, um dann anschließend die gegenseitige Wirkung der auf die Postposition bezogenen synchronen Verbindungen vorzustellen, die gegenseitig Veränderungen indiziert haben.

Die Form át erscheint als Präfix ab Ende der 1830-er Jahre immer häufiger: 1838-45: átugordik '(hin)überspringen', 1841: átváltoztathatik 'umgestaltet werden können', 1844: átharapódzik 'überhandnehmen', 1848: átcsillent 'hin/herübermausen', 1849: átúszik 'durchschwimmen', átírás 'Zuschrift', átküld 'überleiten', 1850: átlépik 'übertreten', átlépendố 'mit der Absicht des Übertritts (Part. Pass.)', átléphetik 'darf übertreten', átlépés 'Übertritt', átléphetés 'Übertrittsmöglichkeit', átlépési 'Übertritts- (Adj)', átlépett 'übergetreten' (SzT.). Bis zu den 1840-er Jahren wechseln sich die beiden Formen noch durchgehend ab, da auch Formen mit által oft vertreten sind. Z. B. 1833: általvehetés 'Übertritt', 1835: általfolyó 'durchfließend', általszökó 'durchziehend/schneidend', általváltoztat 'umtauschen/wechseln', általvett 'übernommen', 1837: általláthat 'verstehen können', 1840: általkölcsönöz '(leihweise) übergeben', 1842: általtört 'durchbrochen', 1844: általadat 'übergeben lassen', általadható 'zu (über)liefernd', általjárogat 'öfters herüberkommen', általmos 'hinüberschwemmen (an das jenseitige Ufer)', 1845: általlép '(durch)treten', 1849: általadódott 'übergeben', általvesz 'übernehmen' (SzT.).

Demnach sind die Formen által und át als Präfix bis zur Mitte des 19. Jh. parallele und gleichgestellte Varianten. Das Verhältnis kippt nach 1850 zugunsten von át um. Während által im SzT. ab den 50-er Jahren nur noch in zwei Verben bzw. deverbalen Ableitungen vorkommt, ist át in zwölf Verben belegt. Diese letztere Periode des Formwechsels - der Sieg von át über das Präfix által — verläuft allerdings auffallend schnell, die Zeit der Schwankung 
ist überraschend kurz, die Verdrängung von által findet fast ohne Übergang statt. Um auszuschließen, dass der damalige Sprachzustand nicht einfach durch die eventuellen Mängel bzw. Einschränkungen des SzT. verstellt wurde, unternahm ich eine flüchtige Untersuchung in Schriftwerken von Personen, die damals im literarischen und kulturellen Leben Siebenbürgens eine herausragende Rolle gespielt hatten und deren Werke in ihrem Charakter dem Quellmaterial des SzT. ähnlich sind, so z. B. in der Korrespondenz von Sámuel Teleki oder der beiden Bolyais und im Tagebuch von Sándor Bölöni Farkas, in den Erinnerungen von János Pálffy und, um einen Ausblick außerhalb des siebenbürgischen Sprachgebrauchs zu gewährleisten, in der Korrespondenz Kazinczys, in der Prosa Vörösmartys, in den Briefen Aranys an Petófi sowie im Petőfi-Wörterbuch. ${ }^{4}$ Diese Untersuchungen ergaben Folgendes: In den Briefen Sámuel Telekis zwischen 1760-1822 war die Variante által die ausschließliche Form. In der Korrespondenz der Bolyais kommt bis 1844 nur által, danach allerdings - nach kurzem Schwanken - nur noch át vor. In den Tagebuchfragmenten Sándor Bölöni Farkas' aus 1835-36 ist durch die wenigen Belege ausschließlich által dokumentiert. Die 1835-36 verfassten Erinnerungen János Pálffys zeigen neben einigen Beispielen mit által eine weitgehende Überlegenheit von át. Für die Sprache der Briefe von Kazinczy ist ebenfalls die Variante át charakteristisch, es kommt sogar einmal ált vor, wird aber nicht wiederholt. Die Angaben in Vörösmartys Prosawerk sind sehr vielsagend. In der 1829 erschienenen Übersetzung der Tausend und eine Nacht wird konsequent által benutzt. In seinen 1837 geschriebenen Erzählungen wechseln sich által und át ab, wobei letzteres ein Übergewicht hat. In den Briefen János Aranys an Petőfi aus 1847-49 kommt ausschließlich die Variante át vor. Im Petőfi-Wörterbuch sind 17 Verben mit dem Präfix által gegenüber 131 Verben mit át verzeichnet. Által kommt ausschließlich in seiner Dichtung vor. Aber selbst dort ist át sehr viel charakteristischer, in seiner Prosa ist es sogar die einzige Variante.

${ }^{4}$ Verwendete Ausgaben: Teleki Sámuel és a Teleki-téka. Téka sorozat [Sámuel Teleki und die Teleki-téka. Reihe Téka]. Kriterion, Bukarest, 1976; Bolyai-levelek. Téka sorozat [Bolyai-Briefe. Reihe Téka]. Kriterion, Bukarest 1975; Bölöni Farkas Sándor naplója. Téka sorozat [Tagebuch des Sándor Bölöni Farkas. Reihe Téka]. Kriterion, Bukarest, 1971; Kazinczy Ferenc levelezése. 23. kötet [Korrespondenz des Ferenc Kazinczy, Bd. 23]. Akadémiai Kiadó, Budapest, 1960; Vörösmarty Mihály Összes múvei. 13. kötet [Gesammelte Werke von Mihály Vörösmarty, Bd. 13]. Akadémiai Kiadó, Budapest, 1974; Magyarországi és erdélyi urak. Pálffy János emlékezései. Sajtó alá rendezte Szabó T. Attila [Herren in Ungarn und Siebenbürgen. Memoiren des János Pálffy. Redigiert von Attila Szabó T.] Erdélyi Szépmíves Céh, 1939; Arany és Petôfi levelezése. Téka sorozat [Korrespondenz von Arany und Petôfi. Reihe Téka]. Kriterion, Bukarest, 1972; Petőfi-szótár I. [Petőfi-Wörterbuch I.]. Akadémiai Kiadó, Budapest, 1973. 
Aus alledem folgt: (a) Die Perioden des Formwechsels von által und át spiegeln sich in der Sprache der Literatur genauso wieder wie in den Daten des SzT., mit dem Unterschied, dass die Variante ált bei Kazinczy und Vörösmarty vereinzelt bereits vor 1830 auftaucht. (b) Im Sprachgebrauch von Petófi und Arany herrschte in der Autorensprache át bereits in den 40-er Jahren vor, sein Gebrauch ging also dem alltäglichen Sprachgebrauch etwas voraus. (c) Die Zeit des Wandels fällt also auf die Zeit, als die Spracherneuerung Früchte zu tragen begann, als die Reformzeit sprachlich ausgereift war, auf eine Zeit, in der das sprachliche Bewusstsein in der Entwicklung der Sprache am ausgeprägtesten war. All dies kann nach meiner Einschätzung als Erklärung für die Ursachen und den Ablauf dieses Wandels dienen.

Der außerordentlich schnelle Sieg von át über által ist vermutlich darauf zurückzuführen, dass die Variante vom literarischen Sprachgebrauch bewusst verbreitet wurde. Dass ein solches Bestreben tatsächlich bestand, lässt nicht nur der individuelle Sprachgebrauch der führenden Autoren erahnen, die den literarischen Sprachgebrauch unmittelbar formten - am meisten vielleicht Vörösmarty; es lässt sich auch durch das erste normative Wörterbuch des Wortgebrauchs, das vorwiegend unter Vörösmartys Mitwirkung erstellte, von der Ungarischen Wissenschaftlichen Gesellschaft 1838 herausgegebene Magyar és német zsebszótár I. Magyar-német rész (Ungarisch-deutsches Taschenwörterbuch) nachweisen. Zum Zeitpunkt seines Erscheinens war, wie das SzT. zeigt, der Kampf zwischen den beiden Varianten in der Alltagssprache längst noch nicht entschieden. Das Ungarisch-deutsche Taschenwörterbuch spiegelt jedoch nicht diesen Sprachzustand wider, sondern sozusagen richtungsweisend den, der erst in den 1850-er Jahren zur sprachlichen Wirklichkeit wird; das Wörterbuch führt nämlich insgesamt nur 15 Formen mit által an, die Zahl der Verben mit át beträgt hingegen 312. Dieses normativ ausgerichtete Wörterbuch legt es also deutlich darauf an, die Variante át gegenüber által zu popularisieren, wobei seine Autorität und allgemeine Verbreitung sicherlich eine Wirkung gehabt haben müssen. Im Falle von át hat die Literatursprache nicht das Ergebnis eines in der spontanen Sprachentwicklung bereits zu Ende gekämpften Kampfes angenommen, vielmehr hat sich der Kampf - anscheinend - durch die Stärke der literarischen Verwendung zugunsten von át entschieden.

Für die weitere Frage, warum die Variante át bevorzugt wurde, warum által seinen präfigalen Status zuerst im literarischen, dann im alltäglichen Sprachgebrauch verloren hat, können zwei Erklärungen herangezogen werden. Die eine ist die Vorliebe für kurze Wortformen, die sowohl im spontanen Sprachgebrauch als auch in den ästhetischen Bestrebungen der Spracherneue- 
rer verbreitet war. Die andere ist die Bestrebung nach Distanzierung bzw. Unterscheidbarkeit von der ähnlichen Postposition.

Die semantisch-funktionelle Einschränkung der postpositionalen Formvarianten wird sich nämlich erst vollzogen haben, nachdem der Tausch der Formvarianten im Präfix bereits weit fortgeschritten war. Által, das auch als Postposition als Hauptvariante galt, kommt nämlich nicht nur in instrumentaler, sondern auch in lokaler und temporaler Funktion überaus häufig im Material des NySz. wie auch des SzT. während des 16-18. Jahrhunderts vor. Die formale Unterscheidung der Funktionen hat scheinbar nicht bei den Formvarianten, sondern bei dem Grundwort der postpositionalen Verbindung angefangen. Während sich der frühere Sprachgebrauch durch den funktional weniger differenzierten Gebrauch von Grundwörtern mit oder ohne Flexionsendung charakterisieren ließ (einem unsuffigierten Grundwort konnte jede beliebige Funktion zugeordnet werden, in lokaler Funktion konnten sich Suffixlosigkeit und die Flexionsendung $-n$, in temporaler Funktion Suffixlosigkeit und das Flexiv - $t$ abwechseln), konnte által ab der zweiten Hälfte des 18. Jh. mit einem unsuffigierten Grundwort nur noch in instrumentaler Funktion stehen. Die Kurzform hatte auch als Postposition zunächst die Form ált und war im 16-18. Jh. wohl äußerst selten. Ihr erster bislang bekannter Beleg zeugt von temporaler Funktion: 1519: „melly collecta mynd eztendew alth mondatyk“ (LányiK. 162) 'welches Kollektengebet im ganzen Jahr gesprochen wird'. Es ist auffällig, dass die Variante át in der Rolle einer Postposition auch in dem reichen Material des SzT. aus dem 19. Jh. nicht belegbar ist. Obwohl diese Postposition in den herangezogenen literarischen Quellen in einer abstrakteren Funktion sehr häufig vorkommt, nimmt sie keine lokale oder temporale Funktion an. Eine begründete Schlussfolgerung ist daher nur anhand des Petôfi-Wörterbuches möglich: nach ihm kommen die Varianten által und át in den 1840-er Jahren in lokaler und temporaler Funktion abwechselnd vor, wobei át bereits stärker vertreten ist (át: 18 lokale, 9 temporale Belege; által: 4 lokale, 2 temporale Belege). Obwohl hier noch weitere sprachgeschichtliche Belege herangezogen werden müssten, kann anhand des Gesagten mit einiger Gewissheit so viel gefolgert werden, dass sich die formale Spaltung der Postposition nach der Verteilung der Funktionen kaum eher als im 19. Jh. abgespielt hat und somit ungefähr auf dieselbe Zeit wie der Formwechsel der präfigalen Varianten fiel. Es ist auch sehr wahrscheinlich, dass die zwischen ihnen bestehende formale und gewisse funktionale Analogie die Herauskristallisierung der Verteilung der Formvarianten gegenseitig begünstigt hat.

Für den funktionellen und semantischen Bereich des Präfixes ist charakteristisch, dass die große Mehrheit der Verben mit által, später mit át einen 
lokalen bzw. temporalen Bezug haben, und nur ein sehr viel kleinerer Anteil dieser Verben übertragene Bedeutung haben kann. Die Stabilisierung der Variante át als Präfix kann in zweierlei Richtungen gewirkt haben: zum Einen erfolgte die vollständige Verdrängung der präfigalen Variante által aus der geschriebenen, später auch aus der gesprochenen Standardsprache. Zum Anderen wurden die verschiedenen postpositionalen Varianten zu formalen Mitteln diverser grammatischer Funktionen gemacht. Da át als Präfix in erster Linie die Richtung oder Zeit einer Tätigkeit oder eines Geschehens bezeichnete, behielt es auch als Postposition dieselbe Variante zum Ausdruck der lokalen und temporalen Funktion bei.

Dass die konkretere Funktion mit át, die abstraktere mit által assoziiert wurde, kann durch einen weiteren systemischen Zusammenhang begünstigt worden sein: durch által in der Agens-Rolle mit der Bedeutung 'mit Hilfe, unter Mitwirkung' sowie durch adverbiale Ableitungen, z. B. általjában átaljában 'im Ganzen' (NySz., SzT.), általában 'zugleich', altung. átalján általán 'im Allgemeinen', általánfogva általánfogván 'durchaus (nicht), im Allgemeinen' (NySz., SzT.), általánvéggel 'ganz gewiss', általképpen 'im Allgemeinen' (NySz.), die ebenfalls eine abstrakte Funktion annahmen bzw. annehmen. Die eher konkrete Bedeutung hat sich im Falle der längeren Formvariante in der Literatursprache von der grammatischen auf die lexikalische Ebene verlagert: sie lebt in Komposita wie általút 'Querweg', általvetố 'Gewand zum Umhängen' weiter.

Die Geschichte der mit den Postpositionen in engem Zusammenhang stehenden Präfixe - wie z. B. által át-beweist, dass ihr Wandel über ihre Entstehungsgeschichte hinaus auch später durch die bestehenden formalfunktionalen Zusammenhänge gesteuert wird.

4. Anhand der Veränderungen im Bereich der Formen des temporalen Gebrauchs mind 'ganz; alle' kann die Wirkung von Systembeziehungen zwischen mehreren Elementen eines Teilsystems beobachtet werden, insbesondere in der Geschichte des Adverbienpaars mindé(l)tig 'immer' und mindig 'ds.'. Zwischen diesen beiden Adverbien hat in den letzten Jahrhunderten der ungarischen Sprachgeschichte eine so enge synchrone Beziehung bestanden, dass sie zeitweise sogar als identisch angesehen wurden (vgl. in $\mathrm{CzF}$. die Wörterbuchartikel mindétig, mindéltig, mindig), und in der früheren etymologischen Literatur wurde mindig ebenfalls aus mindéltig hergeleitet (Simonyi 1888-1892, 357; Horger 1937, 247-249; Beke 1947, 72; SzófSz.). Das TESz. hält dies jedoch für unwahrscheinlich, nach ihm ist mindig eine erstarrte suffigierte Ableitung aus mind 'alle; ganz'. 
Die Ursachenkette, die zur zeitweisen Identifizierung der beiden Adverbien und später zur schnellen Verbreitung von mindig gegenüber mindé(l)tig geführt hat, ergibt sich aus den morphologischen und funktional-semantischen Beziehungen zwischen mindé(l)tig und mindig untereinander sowie aus ihren Beziehungen zu den ihnen am nächsten stehenden Elementen des Adverbiensystems, insbesondere zu mind.

Mindéltig entstand am Anfang der späten altungarischen Zeit, als sich sehr viele zusammengesetzte temporale Adverbien bildeten, besonders häufig unter ihnen waren Verbindungen mit dem Bestimmungswort mind (TNyt. II/1, 615, $626,648)$. Morphologisch-syntaktisch betrachtet wird die primäre Formvariante mindéltig aus einem subordinierenden Kompositum zu einem lexikalisierten Adverb, und zwar aus der vorausgehenden Struktur mind éltig, die dem derzeit bereits vorhandenen komplexen Wortarten- und semantischen Charakter von mind nach (Hámori 1957, 142-47) vermutlich die Bedeutung 'sein ganzes Leben lang', 'ganz bis zum Ende/Schwund seines Lebens', 'in seinem Leben ständig andauernd' hatte.

Zusätzlich zu der ursprünglichen Form mindéltig entstand durch die Elision von /1/ die Variante mindétig. (Ihr erstes sicheres Vorkommen ist 1659 SzT. Die im TESz. angegebene Form vom Anfang des 16. Jh. ist aufgrund eines Schreibfehlers ungewiss.)

In der durch /1/-Elision entstandenen Formvariante wurde die Beziehung des Adverbs zum Grundwort des zweiten Bestandteils, dem Verb él 'leben', offenbar zunächst lockerer, später aufgehoben, so dass im Bestimmungswort die morphologische Struktur vollkommen verblichen ist: mindétig hat sich von dem suffigierten Nomen éltig - éltéig 'solange sein Leben (andauert)' abgelöst und trug somit dazu bei, dass das semantische Merkmal 'Lebensdauer' in einem Teil der adverbialen Bedeutungen wegfiel.

Die historischen Belege (vgl. dazu die Artikel mindéltig in NySz. bzw. SzT.) weisen das Vorhandensein folgender Bedeutungen im 16. Jh. nach. 1. 'ganz bis zum Ende seines Lebens', 2. 'in seinem Leben andauernd', 3. 'zu jeder Zeit', 4. 'in jedem Fall', 5. 'immer, dauernd'.

Während des 17-19. Jh. bestehen die Bedeutungen bezüglich der Lebensdauer (1-2) zwar fort, die andere Bedeutungsgruppe (3-5) jedoch, die das semantische Merkmal des Lebens nicht beinhaltet - die durch Bedeutungserweiterung gebildeten 'zu jeder Zeit' und 'in jedem Fall' bzw. die Bedeutungen 'immer, ständig, andauernd' - , wurde insgesamt dominanter. Dies wurde vermutlich dadurch verstärkt, dass die Bedeutungen 1-2 durch die morphologische Verdunkelung weiter geschwächt wurden. Trotzdem sind in diesen und in allen anderen Bedeutungen das morphologisch und semantisch klar gegliederte 
mindéltig und das morphologisch und semantisch gleichermaßen undurchsichtige mindétig parallel vorhanden. Eine Bedeutungsteilung zwischen den Formvarianten - wie man sie erwarten würde - ist nicht eingetreten.

Es zeigt sich auch durch lexikographische Auslegungen, die ab dem 17. Jh. zur Verfügung stehen, dass die Bedeutungen 3-5 zu diesem Zeitpunkt bereits typischer sind. Beispiele: MA. Perpetuo mind eltig, ô ro kke; PP. mind éltig Perpetuo, Perenne; PPB. Mind étig Perpetuo, Perenne, immerdar, stets, immerwahrend; $\mathrm{CzF}$. Mindétig. „Egyébiránt közönségesen mindig helyett használják; 1. Mindig [Im Übrigen wird es allgemein statt mindig gebraucht; vgl. Mindig]". Demnach ist bis ins 19. Jh. im Bedeutungswandel von mindéltig - mindétig mit Beibehaltung der gesamten Bedeutungsskala die Bedeutung 'andauernd, ständig, immer' allmählich in den Vordergrund gerückt.

Das Adverb mindig mindég ist wesentlich jünger als mindé(l)tig (erstes Vorkommen 1659 SzT., 1772 TESz.). Sein Ursprung, seine Form- und Bedeutungsgeschichte, darunter auch seine Beziehung zu mindé(l)tig, werden durch das reiche historische Material des SzT. erhellt (vgl. den Artikel mindig). Obwohl mindig im 17. Jh. nur durch eine einzige Quelle belegt ist, existiert es im 18. Jh. bereits in fast jeder seiner Bedeutungen als morphologisch und funktionell stabiles, eigenständiges Adverb, das zu Beginn des neuungarischen Zeitalters bereits an Raum gewonnen hat, sowohl in der Alltagssprache und in der Volkssprache als auch in der Literatursprache (Bessenyei, Kazinczy, Baróti Szabó Dávid, TESz.).

Dennoch wird mindig in die Wörterbücher des 18. Jh. noch nicht aufgenommen, beispielsweise kommt es in keiner einzigen Ausgabe des Wörterbuches von Pápai Páriz vor. Die Variante mindég erscheint zuerst in der ersten, 1800 herausgegebenen Auflage des ungarisch-deutschen Wörterbuches von József Márton, in der Bedeutung 'zu jeder Zeit, immer'. Ab diesem Zeitpunkt ist seine lexikographische Präsenz ununterbrochen. Besondere Beachtung verdient seine Aufnahme in das Ungarisch-deutsche Taschenwörterbuch der Ungarischen Wissenschaftlichen Gesellschaft (= Tzs., 1835, 1838), das sich die Entwicklung von Normen für den ungarischen Wortgebrauch sowie die Abgrenzung des Wortschatzes der Literatursprache zum Ziel gesetzt hat und in dieser Hinsicht als Vorläufer des CzF. angesehen werden kann (Gáldi 1957, 489).

Die historischen Belege zeugen von der Überlegenheit der Variante mindég, selbst gegen Mitte des 19. Jh. Die Form mindig ist seit den 80-er Jahren des 18. Jh. belegt (SzT.) und breitet sich allmählich in den ersten Jahrzehnten des 19. Jahrhunderts aus, parallel mit ihm taucht jedoch auch immer die Variante mindég auf. Auch in der Sprache der Literatur lässt sich diese Schwankung beobachten (vgl. NySz. II 840, TESz., Petôfi-Wörterbuch). Der Kampf der beiden 
Formvarianten erscheint in der Praxis der Wörterbuchautoren unterschiedlich beurteilt. Das Tzs. und das CzF. erachten jedoch die Form mindig als geeigneter für die literatursprachliche Norm (wobei sie die größere Häufigkeit von mindég anerkennen).

Von den Bedeutungen mindig mindég taucht als erste 'ständig, fortwährend' auf $(1659,1771)$, aus den letzten beiden Jahrzehnten des 18. Jh. lassen sich jedoch bereits folgende Bedeutungen nachweisen: 'jeder Zeit' (1781), 'in jedem Fall' (1782), 'die ganze Zeit' (1794).

Die Bedeutungsdifferenzierungen 'von einem vergangenen Zeitpunkt bis zur Gegenwart' (1828) bzw. 'sein ganzes Leben lang' (1815) dürften sich - in den entsprechenden Kontexten - bereits mit der Entstehung dieses Adverbs herauskristallisiert oder evtl. später aus den Bedeutungen 'zu jeder Zeit' bzw. 'die ganze Zeit' entwickelt haben. Zur Zeit lassen sie sich für die ersten Jahrzehnte des 19. Jahrhunderts belegen.

In die erste lexikographische Erläuterung - in Mártons Wörterbuch wurde hingegen nur eine der Bedeutungen: 'immer, semper, allezeit' aufgenommen, im Tzs. wird zusätzlich die Bedeutung 'stets' angegeben. Das CzF. gibt eine allgemeine Bedeutungsbestimmung unter Zusammenfassung der Teilbedeutungen an, wobei die Bedeutung 'andauernd, ständig, die ganze Zeit' hervorgehoben wird.

Anhand seiner klaren morphologischen Struktur ist mindig zweifellos eine Ableitung aus mind durch das Flexiv -ig/-ég. Dies wird zuerst im TESz. verlautbart: „Erstarrte suffigierte Ableitung: sie entstand aus dem Pronomen mind durch das Flexiv -ig/-ég, vgl. addig 'bis dort', meddig 'bis wo'. Nach der Entstehung von mindig wurde der Gebrauch von mind in ähnlicher Bedeutung ... etwas eingeschränkt." Diese Herleitung kann in ihrer Essenz, aber nicht in ihren Details akzeptiert werden. Unserer Ansicht nach ist die Entstehung von mindig durch funktionale und systemische Ursachen zu erklären, die jedoch nicht mit dem Pronomen mind, sondern mit dem Adverb mind zusammenhängen. Die Entwicklung von mind als Wortklasse und seine semantische Entwicklung von mind wurde von Antónia Hámori ins Detail gehend erläutert. Demnach wissen wir, dass mind durch eine mehrfache Funktionsentwicklung seit ca. dem 16. Jh. zu einem temporalen Adverb in der Bedeutung 'fortwährend, dauernd, immer' geworden ist (ebda. 147). Neben der temporalen Rolle hat mind jedoch auch seine weiteren adverbialen Funktionen beibehalten, sie wurden durch die neuen Funktionen nicht abgelöst, sondern lebten weiterhin fort, so dass die adverbialen Rollen von mind ebenfalls breit gefächert waren, nicht zu sprechen von seinen weiteren: pronominalen, (paarig) konjunktionalen Verwendungen. Die im 16. Jh. bereits stark ausgeprägte enorme funktionale Belastung von mind 
machte die formale Isolierung von zumindest einer der Funktionen notwendig. Mindig mindég kann also anfangs eine morphologische Variante zur Bezeichnung der temporalen Bedeutung von mind gewesen sein, also mind mindig 'fortwährend, dauernd usw.'. Die neue morphologische Variante entwickelte sich mit dem seit dem späten Altungarischen häufigsten Deklinationssuffix der temporalen Adverbien, angestoßen durch das zu diesem Zeitpunkt stark im Ausbau befindliche System der temporalen Adverbien.

Seine Verstärkung, seine Entwicklung aus einer morphologischen Variante zu einem eigenständigen Adverb wurden durch die systemischen formalen Beziehungen begünstigt: die Adverbien mit dem Suffix -ég/-ig (alig 'kaum', ideig 'eine Zeitlang', késốig 'bis spät', félig 'zur Hälfte', fogytig 'bis zum „Ausgehen", bis zum Schluss', váltig 'bis zum Scheiden', vég(ezet)ig 'bis zum Ende'), aber vor allem die Zusammensetzungen mit mind (mindeddig 'ganz bis jetzt', mindaddig 'ganz bis dahin', mindmáig 'ganz bis heute', mindvégig 'ganz bis zum Schluss', mindéltig 'in seinem ganzen Leben', mindholtig 'bis zum Tod'), in deren Reihe sich mindig auf natürliche Weise einfügen konnte. Und damit konnte seine Ablösung von seinem Ausgangswort einsetzen: vom in seiner morphologischen Struktur nicht temporalen und an anderen, nicht temporalen Funktionen ebenfalls reichen mind.

Das Adverb mindig hatte durch das vom bedeutungsreichen mind geerbte breite Bedeutungsfeld einen Vorsprung gegenüber anderen partiellen Synonymen, die nur ein semantisches Merkmal mit ihm gemeinsam hatten und die parallel zur Verbreitung von mindig ganz oder allmählich aus dem Sprachgebrauch verdrängt wurden, wie z. B. mindenha 'allemal/zeit', mindenszer 'jedesmal'.

In engster Synonymie stand das Adverb mit mindéltig mindétig. Im 1819. Jh. deckten sich ihre Bedeutungen fast vollständig, außer dem Bezug auf die Lebensdauer, die zu mindig ursprünglich nicht gehört hatte. Als Folge der verblichenen Morphologie geriet diese Bedeutung jedoch auch in mindétig in den Hintergrund, und ab dem 18. Jh. - als diese Variante die üblichere war wurde diesem Adverb ebenfalls die Hauptbedeutung 'jeder Zeit, in jedem Fall' zuteil. Zu diesem Zeitpunkt enthielt mindig neben der Bedeutung 'andauernd, ständig' auch schon diese Bedeutungen. Die anfängliche partielle Synonymie der beiden Adverbien wurde somit zu einer vollständigen. Die phonologische Ähnlichkeit war ebenfalls am stärksten zwischen dem mittlerweile zur Hauptvariante gewordenen mindétig und dem damals noch als Hauptvariante geltenden mindég. Die vollständige Synonymie und die phonologische Ähnlichkeit der beiden Adverbien kann zu ihrer vollkommenen Identifizierung geführt haben. Dies lässt sich anhand der Wörterbücher aus dem 19. Jh. eindeutig bestätigen: mindétig und mindig werden als Varianten desselben Wortes betrachtet. Bei 
der Entwicklung der standardsprachlichen Normen wird für das „regelhaftere“ mindig entschieden, da es sich besser in die Reihe der deklinierten temporalen Adverbien einordnen lässt. Die Variante mindétig hingegen wird von der Norm ausgeschlossen und gilt mit der Expansion der Literatursprache allmählich als veraltet bzw. lebt nur noch dialektal weiter. Mindig hingegen wird in die Norm aufgenommen, was zur Folge hatte, dass es in einer für Adverbien ungewöhnlich kurzen Zeit Akzeptanz fand und mindétig verdrängte.

Gemeinsam für die dargestellten Wandelphänomene ist, dass Wirkungen aus den unterschiedlichen Sprachschichten die morphonologischen, semantischen und funktionalen Beziehungen zwischen bestimmten Elementen/Teilbereichen der Sprache so stark beeinflussen (enger oder lockerer machen), dass sie von ihrer früheren Position weggerückt werden, d. h. ihr früherer Charakter modifiziert bzw. verändert sich in eine durch derartige Beziehungen bestimmte Richtung.

\title{
Literatur
}

Bárczi, Géza 1958. Magyar történeti szóalaktan. I: A szótövek [Historische Wortmorphologie des Ungarischen, I: Wortstämme]. Tankönyvkiadó, Budapest.

Beke, Ödön 1947. Eddig, mindig [Bislang, immer]. In: Magyar Nyelvôr $71: 72$.

Gáldi, László 1957. A magyar szótárirodalom a felvilágosodás korában és a reformkorban [Ungarische Wörterbuchliteratur in der Zeit der Aufklärung und während der Reformzeit]. Akadémiai Kiadó, Budapest.

Hámori, Antónia 1957. Mind [Alle]. In: Magyar Nyelv $52: 138-47$.

Horger, Antal 1937. Mindig [Immer]. In: Magyar Nyelv 33 : 247-49.

Simai, Ödön 1910. Márssal Társalkodó Murányi Vénus [Venus von Murány, im Gespräch mit dem Mars]. In: Magyar Nyelv 6:162-66.

Simonyi, Zsigmond 1888-1892. A magyar határozók I-II [Ungarische Adverbien I-II]. Magyar Tudományos Akadémia, Budapest.

\author{
Adresse der Verfasserin: Piroska B. Gergely \\ Universität Miskolc \\ Institut für Ungarische Sprachwissenschaft \\ H-3515 Miskolc-Egyetemváros \\ Ungarn \\ bolpiko@gold.uni-miskolc.hu
}

Acta Linguistica Hungarica 49, 2002 